\title{
The life of Hans-Günter Neumann and his contributions to chemical carcinogenesis
}

\author{
Manfred Metzler ${ }^{1} \cdot$ Annette Bitsch ${ }^{2} \cdot$ Gisela H. Degen ${ }^{3}$
}

Received: 4 March 2020 / Accepted: 10 March 2020 / Published online: 16 March 2020

c) Springer-Verlag GmbH Germany, part of Springer Nature 2020

\section{Introduction}

With the death of Professor Hans-Günter Neumann, the scientific community has lost an outstanding and passionate scientist whose contributions to carcinogenesis of aromatic amines and biomonitoring have been pioneering. Günter passed away on November 05, 2019, in his home in Würzburg, Germany, at the age of 86 . His last period of life was burdened with illness, but he had been provided with loving care by his wife Monika and sons Christian and Andreas and their families.

Günter was born in Munich in 1933. He received a Diploma in Chemistry from the University of Munich in 1959 and earned a Ph.D. in Chemistry in 1961 with a dissertation on the dehydrogenation of steroids, conducted at the Max-Planck-Institute of Biochemistry in Munich. During a 1-year postdoctoral period in Elwood Jensen's group at the Ben-May-Laboratory for Cancer Research of the University of Chicago, Günter used his chemistry skills to synthesize radiolabeled estrogenic steroids of very high specific radioactivity. He was involved in Jensen's studies on the detection of the estrogen receptor and metabolism of contraceptive steroids. The beginning of his scientific career and development of his further research has been described by Günter himself after his retirement (Neumann 2004).

Gisela H. Degen

degen@ifado.de

Manfred Metzler

manfred.metzler@kit.edu

Annette Bitsch

annette.bitsch@item.fraunhofer.de

1 Institute of Applied Biosciences, Karlsruhe Institute of Technology (KIT), Karlsruhe, Germany

2 Fraunhofer-Institut für Toxikologie und Experimentelle Medizin (ITEM), Hannover, Germany

3 Leibniz-Institut für Arbeitsforschung an der TU Dortmund (IfADo), Dortmund, Germany
After his return from Chicago to Munich, he applied the strategy of high radiolabelling to carcinogenic amines, many of which exhibit pronounced organ specificity. The reasons for this organotropic carcinogenicity of aromatic amines were unknown and thought to reside either in accumulation of the carcinogenic agent or in a higher level of DNA adducts in the target organ. Yet, data in support of this view or their dose-dependent kinetics were not available at that time. Aromatic amines were known to undergo metabolic activation to various $\mathrm{N}$-oxidation products (Fig. 1). trans4-Dimethylaminostilbene (trans-DAS), a powerful and selective carcinogen for the "Zymbal's gland" (a sebaceous gland in the ear duct of rats), and two non-carcinogenic congeners, i.e., the cis-isomer (cis-DAS) and the hydrogenation product 4-dimethylaminobibenzyl (DABB), were the perfect model amines to address the puzzle of organotropism, because these compounds were suitable for high radiolabeling (Fig. 1). The label allowed elucidating the distribution, metabolism, and covalent binding to tissue macromolecules down to extremely low doses in rats. Later in his work, Günter expanded the group of model compounds within the aromatic amines with trans-4-acetyl-aminostilbene, 2-acetylaminofluorene and 2-acetylaminophenanthrene, still focusing on the complex mechanisms prompting the cancer development in specific target organs (see below).

\section{Mechanistic studies on organotropism and dose-response relationships}

The studies with trans-DAS, cis-DAS and DABB were started in Munich and continued at the Institute of Toxicology and Pharmacology of the University of Würzburg, which Günter joined in 1971. At carcinogenic doses of radiolabeled trans-DAS, the highest levels of radioactivity were observed in rat liver and kidney, whereas the radioactivity found in Zymbal's gland after trans-DAS was about the same as in other lipophilic tissues such as fat, adrenals, or lymph 
Fig. 1 Simplified scheme of the metabolism of aromatic amines, together with the aryl compounds used in Günter's studies, and the cellular targets of reactive metabolites. The position of the amino group (numbered $\mathrm{C}$-atom of the aryl moiety) and of the radiolabel is indicated. RO may be acetate or sulfate

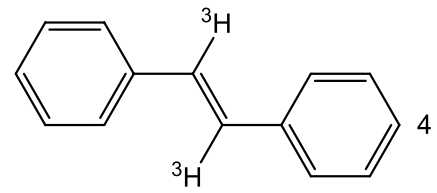

trans-stilbene<smiles>c1ccc2c(c1)Cc1ccccc1-2</smiles>

fluorene

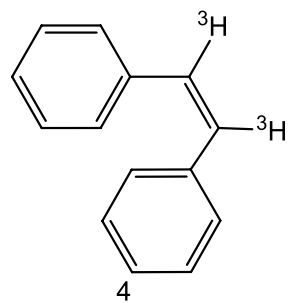

cis-stilbene

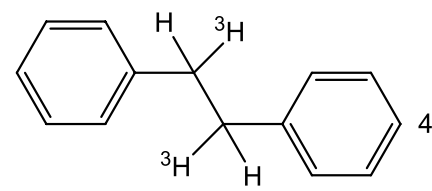

bibenzyl

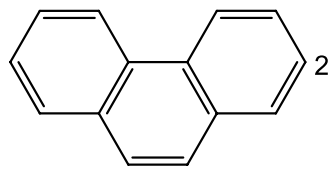

phenanthrene<smiles>CCN(C)C</smiles><smiles>[Li]NC=C</smiles><smiles>CC(=O)NC=O</smiles>

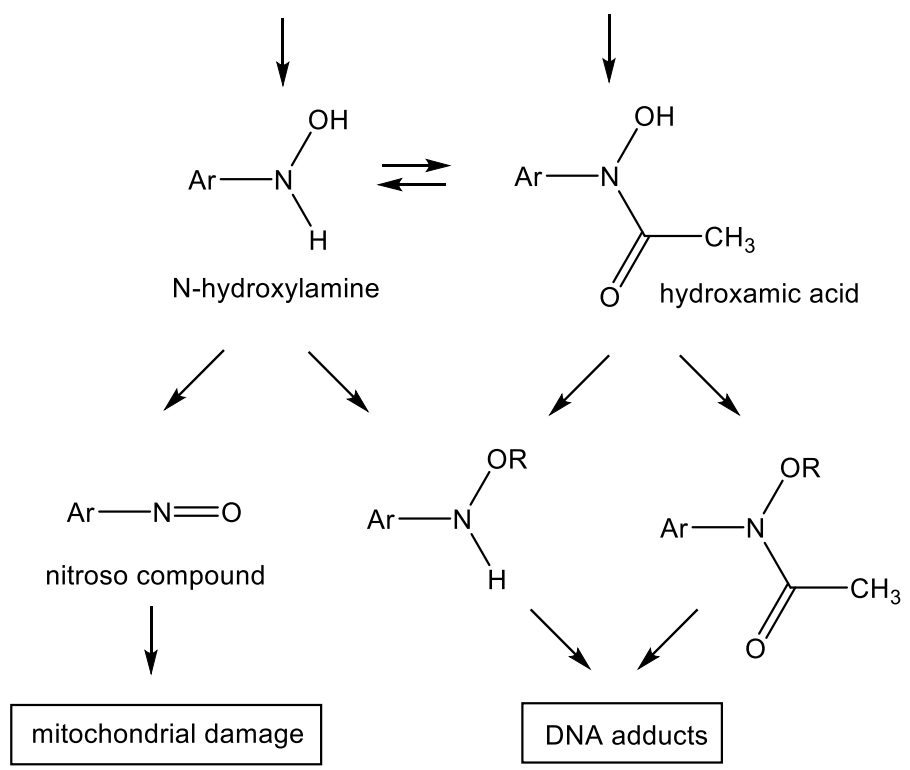

$\mathrm{N}$-hydroxylamine<smiles>[3H]CC[3H]</smiles>

nodes. Moreover, radioactivity in Zymbal's gland was about the same after trans-DAS application as after cis-DAS or DABB application (Rjosk and Neumann 1971). This clearly showed that neither the parent carcinogen nor its metabolites accumulate in the target organ. What then about levels of DNA adducts in Zymbal's gland compared to non-target organs? Although the highly radiolabeled trans-DAS did not allow to directly measure DNA adducts in the tiny Zymbal's gland, several other lines of evidence eventually suggested that the presence of DNA adducts in the target organ was necessary, but not a sufficient prerequisite to explain the organotropic action of trans-DAS. This indicated-in line with the multistage concept of carcinogenesis-that the final adverse effect (cancer) is not based on genotoxicity alone, but additional consecutive biological processes are required.
Among the important results of such pioneering studies in the 1970s was the finding that the distribution, metabolic activation and covalent macromolecular binding of carcinogens follow first-order kinetics even at extremely low doses (reviewed in Neumann 2009). In the respective study, radiolabeled trans-DAS was orally administered at doses covering six orders of magnitude, ranging from a high (nearly lethal) to 100 -fold below the carcinogenic dose. Notably, the extent of covalent binding of radioactivity to DNA, RNA and proteins in all organs measured (liver, kidney, stomach and blood) proved to be proportional to the dose even at the lowest dose of $25 \mathrm{ng}$ trans-DAS per rat. Given that DNA adducts are promutagenic lesions, this observation clearly showed that a threshold of genotoxicity cannot be defined for the carcinogenic aromatic amine (Neumann 1980). This 
conclusion was later supported by other laboratories working with various DNA-reactive chemical carcinogens. Günter's work furthermore demonstrated that the ultimate reactive metabolite of trans-DAS, i.e., $N$-acetoxy- $N$-acetyl-trans4-aminostilbene, after oral administration to rats also caused exclusively tumor growth in the Zymbal's gland, despite the fact that DNA adducts were generated in every organ. This again suggested that factors other than DNA damage must be involved in organotropism. It was suspected that the high proliferative activity of this gland, due to disruption of cells during release of the tallow, makes it vulnerable.

Understanding the organotropism of aromatic amines remained the major interest in Günter's further research. As DNA adducts were formed in every organ following metabolic activation, liver and kidney appeared to be suitable for further studying the importance of cell proliferation. Indeed, trans-4-acetyl-aminostilbene (trans-AAS), a metabolite of trans-DAS (Fig. 1) and not a liver or kidney carcinogen per se, gave rise to hepatic tumors in rats when liver cell proliferation was stimulated by partial hepatectomy or certain liver tumor promotors such as phenobarbital, the insecticide DDT or the synthetic estrogen diethyl-stilbestrol (Neumann 1983). This approach already considered the multistage concept of carcinogenesis (Scott et al. 1984) suggesting that initiation is the process of introducing irreversible lesions in genomic DNA, whereas the promotion regimen enables proliferation and accumulation of preneoplastic cells. Similarly, renal tumors were observed when rats were dosed with transAAS and subsequently treated by unilateral nephrectomy and given nephrotoxic antibiotics. Further studies focused on the liver and the three aromatic amines trans-AAS, 2-acetylaminofluorene (AAF) and 2-acetylamino-phenanthrene (AAP, Fig. 1). These compounds are carcinogens with different organotropism in rats: trans-AAS in Zymbal's gland, AAF in liver, and AAP in mammary gland. In cultured mammalian cells, all three aromatic amines were shown to be mutagenic after metabolic activation. Their mutagenic potency was in a similar range and even the type of mutations was similar, showing a focus on $\mathrm{G}$ to $\mathrm{T}$ transversions and $\mathrm{G}$ to $\mathrm{C}$ transitions. Moreover, all three amines gave rise to liver tumors in newborn rats receiving phenobarbital drinking water as promoting agent. The tumor incidence was comparable over lifetime, but there were indications that the onset of tumor formation was earliest for AAS. A large set of investigations were subsequently conducted for a better understanding of the process of tumor formation. This started with the investigation of some typical oncogenes such as H-ras and p53, but also included a complex histopathology for architectural rebuilding of the liver. In the tumor tissue, surprisingly, no mutations of $\mathrm{H}$-ras or p53 could be detected (Bitsch et al. 1993). The two cell growth and differentiation related proto-oncogenes c-FOS and c-JUN, however, were increased in all AAF-treated animals at early stages and even more early in the process of tumor formation the number of apoptotic cells was increased (Hadjiolov et al. 1995; Hadjiolov and Bitsch 1997).

Günter's studies so far had shown that all three model amines were able to generate DNA adducts and mutations in rat liver cells, but only AAF gave rise to liver tumors, whereas trans-AAS and AAP needed additional stimulation of cell proliferation to become tumorigenic. In the terminology of chemical carcinogenesis, all three amines were able to generate initiated cells after metabolic activation, but only AAF was a complete carcinogen, whereas promotion of initiated cells was required in the case of trans-AAS and AAP. What made AAF a complete carcinogen? It was soon noted that AAF, in contrast to the two other amines, was highly toxic for the liver. In a series of systematic studies, it finally turned out that the cellular target of AAF cytotoxicity was the mitochondria and even the molecular mechanism of toxicity could be elucidated. Two of the active metabolites of AAF, i.e., $N$-hydroxy-AF and 2-nitrosofluorene (see Fig. 1) are highly effective in inducing a redox cycle at complex I and complex III of the mitochondrial respiratory chain, thereby impairing mitochondrial respiration and oxidative phosphorylation (Neumann et al. 1997; Klöhn et al. 1998, 2003). Initially, this leads to an opening of the mitochondrial permeability transition pore (PTP) and depolarization of the mitochondrial membrane, followed by nuclear apoptosis and regenerative proliferation leading to cirrhosis-like transformation of liver tissue. However, upon feeding of AAF for 2 weeks or longer, a dose-dependent inhibition of the PTP was observed, accompanied by increased expression of antiapoptotic BCL-2. This was considered an adaptive response of the liver cell to the chemical stress of AAF. The selective suppression of the mitochondrial pro-apoptotic pathway in liver cells early during AAF carcinogenesis enables the persistence of initiated cells and promotes the growth of preneoplastic foci and tumors.

The sequence of molecular and cellular events disclosed in Günter's studies on AAF-induced carcinogenesis can be viewed as an early example of a concept known later as an adverse outcome pathway (AOP). An AOP developed for a specific type of toxicity represents a pathway giving a formalized view on mechanistic toxicological information: AOPs describe a sequence of events, from a molecular initiating event (MIE), followed by several key events (KEs) and transitions that finally lead to a specific adverse outcome. This chemical-agnostic approach linking a set of biological events/responses to apical end points can be also used to design mechanism-based testing methods. For the use in risk assessment, however, the information gathered in an AOP is somewhat incomplete and should be extended by consideration of ADME properties for chemicals in an integrated approach (Leist et al. 2017). As many mutagenic chemicals must undergo metabolic activation from a pro-mutagen to 
the final DNA damaging metabolite, the chemical-specific mode of action part would be incomplete without this knowledge. A preliminary AOP for the carcinogenic effects of AAF based on the results of Günter's work is proposed in Fig. 2.

In summary, Günter's systematic studies aiming at a better understanding of organotropism have shed new light on the importance of non-genotoxic events in the course of tumorigenesis. As genetic damage per se is necessary, but not sufficient for tumor formation, the epigenetic toxic effects of carcinogens eventually determine in which organ tumors develop. This also has a bearing on risk assessment of chemical carcinogens, because linear dose-response relations without threshold have only been demonstrated for DNA-reactive genotoxic agents but not for epigenetic effects (Bitsch et al. 2000; Neumann 2007, 2009, 2010).

Although Günter's research on his key scientific areas of interest, i.e., mechanisms of chemical-induced carcinogenesis and human biomonitoring, involved primarily aromatic nitrogen compounds, his laboratory also worked with other mutagenic carcinogens. For example, the reason for the striking difference in susceptibility of rats and mice to the carcinogenic activity of the mycotoxin aflatoxin $\mathrm{B}_{1}\left(\mathrm{AFB}_{1}\right)$ was disclosed in the 1980s: both species transform AFB $_{1}$ to its 8,9-epoxide as ultimate reactive metabolite, but the less susceptible mouse is far more efficient in conjugating the exo-epoxide with glutathione than the highly susceptible rat (Degen and Neumann 1981). This data showed for the first time that differences in the detoxication ability rather than formation of the reactive metabolite can be crucial for susceptibility to carcinogens. Recent studies confirm the role of subunit glutathione transferase-A3 in protection of adult mice against $\mathrm{AFB}_{1}$ toxicity (Ilic et al. 2010). Also consistent with glutathione transferase activity are differences in mutagenicity and carcinogenicity of $\mathrm{AFB}_{1}$ in neonatal and in adult mice (Chen et al. 2010). Knowledge of $\mathrm{AFB}_{1}$ metabolism and its mechanisms of action enabled the development of molecular biomarkers of exposure and effect which are useful in assessing human exposure to aflatoxins and the modulation of $\mathrm{AFB}_{1}$ disposition by chemopreventive agents in (rodent and human) intervention studies (Kensler et al. 2011). $\mathrm{AFB}_{1}$ - one of the most intensely studied chemicals-served also in the development of an AOP for hepatocellular carcinoma induction by this mutagenic human carcinogen (Moore et al. 2018).

\section{Human biomonitoring: from early findings to application}

Another pioneering area of Günter's research was biomonitoring of aromatic amines, many of which are important environmental or working place contaminants per se or are formed as metabolites, e.g., of nitroaromatic or azo compounds. Interest in this topic developed when the organ distribution of radiolabeled aromatic amines was studied in

Proposed preliminary AOP for the AAF-induced liver carcinogenicity

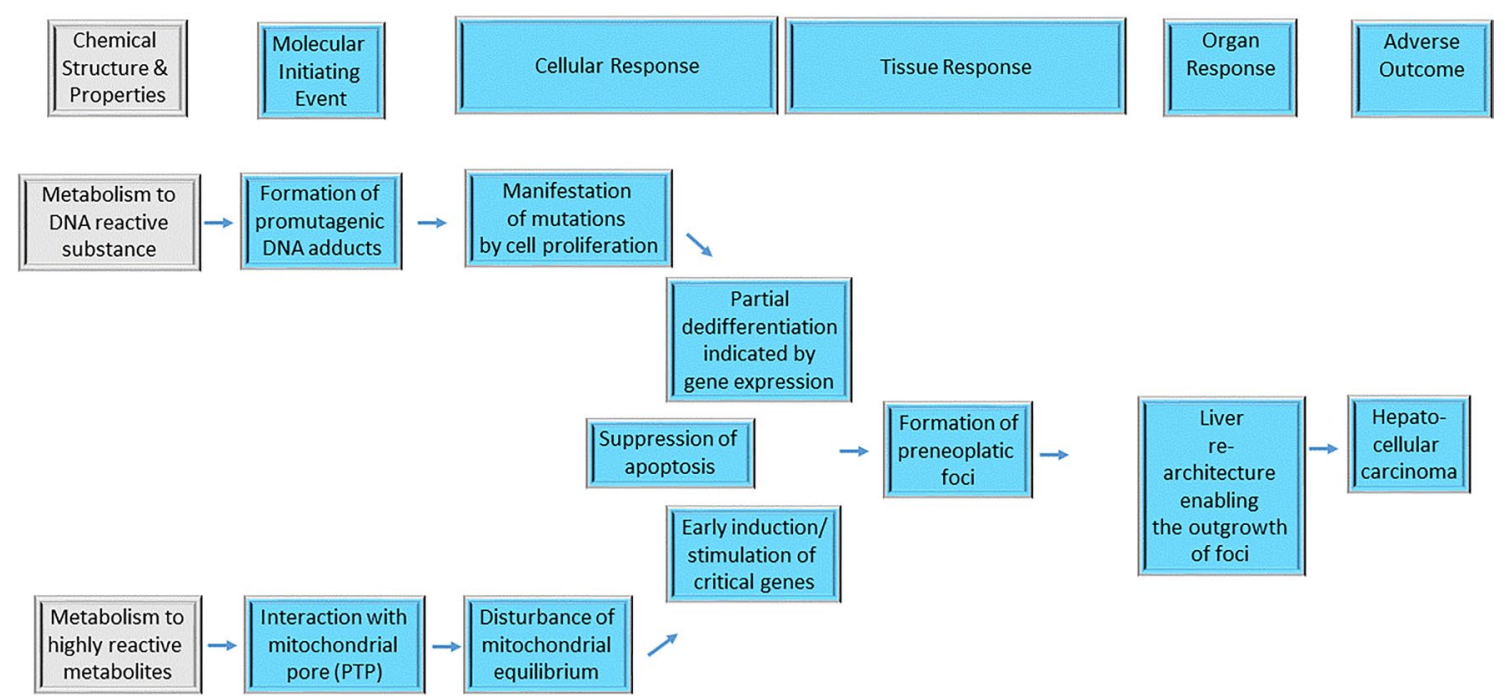

Fig. 2 A preliminary AOP proposal depicting critical steps during AAF-induced liver carcinogenesis based on Günter's work. The two initiating effects are considering the activities of the respective active metabolites, i.e., DNA binding and mutagenicity on one hand and the interaction with the mitochondrial pore (PTP) leading to mitochon- drial imbalance and deregulated gene expression on the other hand. It finally shows the interaction between the DNA damage and the outgrowth in preneoplastic foci as a consequence of liver re-architecture and thus represents more a network than a linear scheme of critical events 


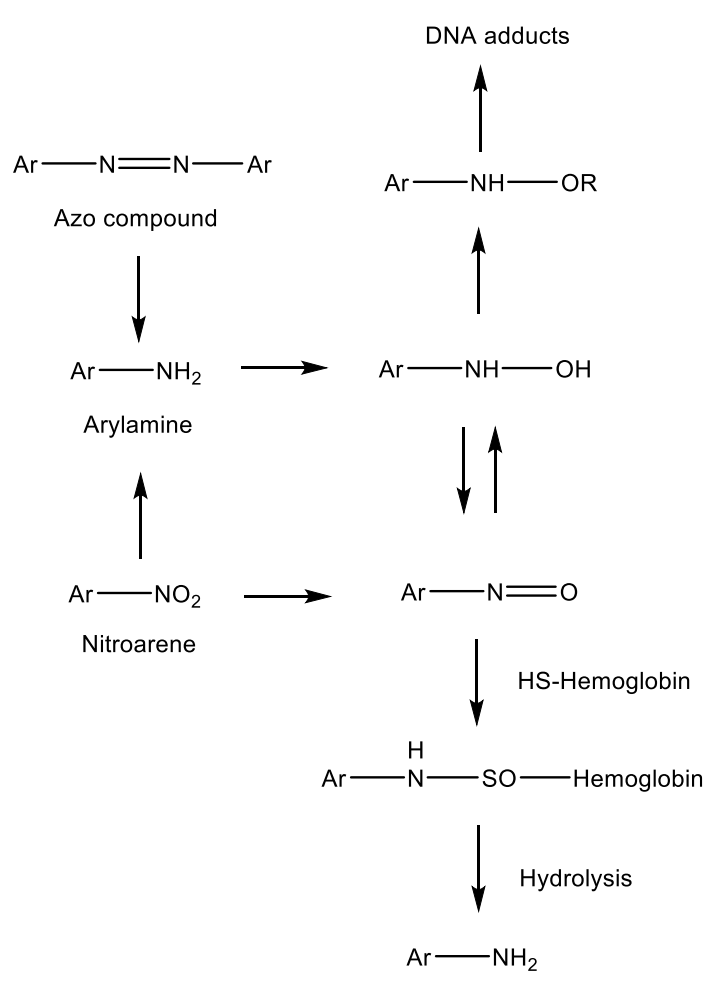

Fig. 3 Simplified scheme of the metabolic activation of azo compounds, amino arenes and nitro arenes and the formation of adducts with DNA and hemoglobin. RO may be acetate or sulfate

the 1970s (see above). The high radioactivity in blood was shown to be due to covalent binding of reactive metabolites to hemoglobin. The binding was soon identified as the reaction of aromatic nitroso metabolites with cysteine moieties of hemoglobin yielding sulfinic acid amides, which upon mild chemical hydrolysis released the amine and were therefore easy to measure (Fig. 3). Günter realized the potential of this reaction for exposure and biochemical effect monitoring and proposed it for human biomonitoring (Neumann 1984). He demonstrated that the hemoglobin adducts were stable in vivo and did not affect the life span of erythrocytes. Moreover, covalent binding of aromatic amines to hemoglobin exhibited the same linear dose dependency as covalent binding to DNA, measured with trans-DAS (see above) over a dose range of five orders of magnitude (Neumann et al. 1993).

Günter not only addressed basic questions of the biomonitoring of aromatic nitrogen compounds, but also used it for molecular epidemiological studies, e.g., on exposure of workers to diesel exhaust or fumes from coke ovens or of people living in former military areas contaminated with explosives. Common findings of such studies were that environmental exposure to monocyclic aromatic nitro compounds is widespread and exposure varies widely between individuals even under very similar living or working conditions (Neumann et al. 1995a, b). Such large interindividual variation in biomarker levels revealed the need to consider the contribution of specific exposures relative to 'background' in the context of risk assessment and risk management. As well reviewed by others (Pathak et al. 2016; Sabbioni 2017) hemoglobin adducts of arylamines and nitroarenes have been used in many human biomonitoring studies for over 30 years, including many fundamental studies of Günter's laboratory. Also adducts of reactive compounds or metabolites with serum albumin are valuable biomarkers of exposure to important carcinogenic food contaminants or drugs (Sabbioni and Turesky 2017). Nowadays, progress in analytical methodologies allows for untargeted screening of albumin and hemoglobin adducts in human blood (Carlsson et al. 2019; Möller et al. 2017). Such 'protein adductomics' will expand our knowledge on human exposure to toxic chemicals, including carcinogenic agents from exogenous and endogenous sources.

\section{Concluding remarks}

Key questions of chemical carcinogenesis addressed in Günter's pioneering studies continue to be relevant research topics for mutagenic carcinogens and mechanism-based risk assessments: his work on the organotropism of aromatic amines reveals that distribution, metabolic activation and covalent binding to DNA follow first-order kinetics even at very low doses. Occurrence of promutagenic DNA lesions in both target and in non-target organs indicates that the induction of tumors requires further steps and processes; these have been elucidated in part for AAF, paving the road now for development of an AOP. Also, Günter's scientific achievements in the field of human biomonitoring have not been forgotten, as exemplified by the recent state-of-the-art review.

Günter retired from the University of Würzburg in 1998 but kept a keen interest in chemical carcinogenesis until the end of his life. Because of his gentle, fair and kind personality, many of his graduate students and research associates over the years became friends of him and kept this relation for a long time. They were all very sad to see this great scientist and remarkable man gone. Fortunately, his contributions to the field of toxicology will live on.

\section{References}

Bitsch A, Röschlau H, Deubelbeiss C, Neumann HG (1993) The structure and function of the $\mathrm{H}$-ras proto-oncogene are not altered in rat liver tumors initiated by 2 -acetylaminofluorene, 2-acetylaminophenanthrene and trans-4-acetylaminostilbene. Toxicol Lett 67:173-186 
Bitsch A, Hadjiolov N, Klöhn PC, Bergmann O, Zwirner-Baier I, Neumann HG (2000) Dose response of early effects related to tumor promotion of 2-acetylaminofluorene. Toxicol Sci 55:44-51

Carlsson H, Rappaport SM, Törnqvist M (2019) Protein adductomics: methodologies for untargeted screening of adducts to serum albumin and hemoglobin in human blood samples. High-Throughput. 8:6. https://doi.org/10.3390/ht8010006

Chen T, Heflich RF, Moore MM, Mei N (2010) Differential mutagenicity of aflatoxin B1 in liver of neonatal and adult mice. Environ Mol Mutagen 51:156-163

Degen GH, Neumann HG (1981) Differences in aflatoxin $\mathrm{B}_{1}$-susceptibility of rat and mouse are correlated with the capability in vitro to inactivate aflatoxin $\mathrm{B}_{1}$-epoxide. Carcinogenesis 2:299-306

Hadjiolov N, Bitsch A, Neumann HG (1995) Early initiating and promoting effects in 2-AAF-induced rat liver carcinogenesis: an immunohistochemical study. Cancer Lett 98:39-46

Hadjiolov N, Bitsch A (1997) Early effects in chemical-induced rat liver carcinogenesis: an immunohistochemical study following exposure to $0.04 \%$ AAF. Apoptosis 2:91-100

Ilic Z, Crawford D, Egner PA, Sell S (2010) Gluthione-S-transferase A3 knockout mice are sensitive to acute cytotoxic and genotoxic effects of aflatoxin B1. Toxicol Appl Pharmacol 242:241-246

Klöhn PC, Bitsch A, Neumann HG (1998) Mitochondrial permeability transition is altered in early stages of carcinogenesis of 2-acetylaminofluorene. Carcinogenesis 19:1185-1190

Klöhn PC, Soriano ME, Irwin W, Penzo D, Scorrano L, Bitsch A, Neumann HG, Bernardi P (2003) Early resistance to cell death and to onset of the mitochondrial permeability transition during hepatocarcinogenesis with 2-acetylaminofluorene. Proc Natl Acad Sci USA 100:10014-10019

Kensler TW, Roebuck BD, Wogan GN, Groopman JD (2011) Aflatoxin: A 50-year odyssey of mechanistic and translational toxicology. Toxicol Sci 120(S1):S28-S48

Leist M, Ghallab A, Graepel R, Marchan R, Hassan R et al (2017) Adverse outcome pathways: opportunities, limitations and open questions. Arch Toxicol 91:3477-3505

Möller C, Clay Davis W, Thompson VR, Mari F, DeCaprio AP (2017) Proteomic analysis of thiol modifications and assessment of structural changes in hemoglobin induced by the aniline metabolites N-phenylhydroxyl-amine and nitrosobenzene. Scientific Reports 7:14794. https://doi.org/10.1038/s41598-017-14653

Moore MM, Schoeny RS, Becker RA, White K, Pottenger LH (2018) Development of an adverse outcome pathway for chemically induced hepatocellular carcinoma: case study of AFB1, a human carcinogen with a mutagenic mode of action. Crit Rev Toxicol 48:312-327

Neumann HG (1980) Dose-response relationship in the primary lesion of strong electrophilic carcinogens. Arch Toxicol Suppl 3:69-77

Neumann HG (1983) Role of tissue exposure and DNA lesions for organ-specific effects of carcinogenic trans-4-acetylaminostilbene in rats. Environm Health Perspect 49:51-58
Neumann HG (1984) Analysis of hemoglobin as a dose monitor for alkylating and arylating compounds. Arch Toxicol 56:1-6

Neumann HG (2004) From chemistry to toxicology—a personal look back (in German). Umweltmed Forsch Prax 9:33-44

Neumann HG (2007) Aromatic amines in experimental cancer research: tissue-specific effects, an old problem and new solutions. Crit Rev Toxicol 37:211-236

Neumann HG (2009) Risk assessment of chemical carcinogens and thresholds. Crit Rev Toxicol 39:449-461

Neumann HG (2010) Aromatic amines: mechanisms of carcinogenesis and implications for risk assessment. Front Biosci 15:1119-1130

Neumann HG, Birner G, Kowallik P, Schütze D, Zwirner-Baier I (1993) Hemoglobin adducts of N-substituted aryl compounds in exposure control and risk assessment. Environ Health Perspect 99:65-69

Neumann HG, Albrecht O, van Dorp C, Zwirner-Baier I (1995a) Macromolecular adducts caused by environmental chemicals. Clin Chem 41(42): 1835-1840

Neumann HG, van Dorp C, Zwirner-Baier I (1995b) The implications for risk assessment of measuring the relative contribution to exposure from occupation, environment and lifestyle: hemoglobin adducts from amino- and nitro-arenes. Toxicol Lett 82-83:771-778

Neumann HG, Bitsch A, Klöhn PC (1997) The dual role of 2-acetylaminofluorene in hepatocarcinogenesis: specific targets for initiation and promotion. Mutat Res 376:169-176

Pathak KV, Chiu TL, Ambrose Amin E, Turesky RJ (2016) Methemoglobin formation and hemoglobin adducts of carcinogenic aromatic amines and heterocyclic aromatic amines. Chem Res Toxicol 29:255-269

Rjosk HK, Neumann HG (1971) The relevance of chemico-biological interactions for the toxic and carcinogenic effects of aromatic amines. II. Distribution of radioactivity in the rat following administration of the tritium-labelled carcinogen trans-4-dimethylaminostilbene and non-carcinogenic cis-4-dimethyl-aminostilbene and 4-dimethylaminobibenzyl (article in German). Z Krebsforsch 75:209-220

Sabbioni G (2017) Hemoglobin adducts and urinary metabolites of arylamines and nitroarenes. Chem Res Toxicol 30:1733-1766

Sabbioni G, Turesky R (2017) Biomonitoring human albumin adducts: The past, the present, and the future. Chem Res Toxicol 30:332-366

Scott RE, Wille JJ, Wier ML (1984) Mechanisms for the initiation and promotion of carcinogenesis: A review and a new concept. Mayo Clin Proc 59:107-117

Publisher's Note Springer Nature remains neutral with regard to jurisdictional claims in published maps and institutional affiliations. 